\title{
Ho-Hum West, No-Nonsense East
}

\author{
Fyodor A. Lukyanov
}

DOI: $10.31278 / 1810-6374-2021-19-2-5-8$

It will soon be twenty years (since 2002) as our journal has been keen to make a modest contribution to the discussion of international relations and encourage dialogue between different countries and strategic cultures. Much has changed over these years. World politics has become quite startling and has acquired features of tragifarce, increasingly fanciful. Classical times, even those of confrontation, can only be reminisced about. The environment has become extremely contradictory: everything and everybody are very closely interconnected, but at the same time do not trust and fear each other. All is strange and it is unclear how to pursue a consistent policy.

Such a transformation of international practices has different consequences; one of them is the emasculation (up to the complete loss of any sense) of political expert dialoguenot among diplomats, officials or statesmen, but among international relations specialists, whose discussions have always accompanied official negotiations. Our authors are among such pundits.

The traditional view is that even in the most hopeless moments of confrontation, it is necessary to maintain dialogue in order to understand each other's attitude and avoid the worst. And this is how it works when world politics is more or less orderly. Unfortunately, we can only dream of that now. In fact, when foreign policy actions are increasingly determined by momentary internal objectives, a rational discussion of a possible detente becomes almost impossible. This primacy of the internal over the external becomes more and more obvious and persistent nowadays. Everywhere.

And yet, attempts to maintain international dialogue do not stop. Two models, known from the recent past, are used as a basis for it. The first one feeds off the legacy of the 
period immediately after the Cold War when it was believed that cooperation was possible only on the basis of a certain set of values, and compliance with them was regarded as a criterion of success. This is primarily the European approach. The second one, advocated by Russian and some American experts, revives the Cold War approach: whatever the differences, there is a common responsibility of the two superpowers for strategic stability and therefore they must find areas for fruitful interaction.

The efficacy of the first model is now quite obvious. A dialogue on an ideological basis was possible (not synonymous with "productive") when all of its participants recognized universal principles. This continued (albeit gradually fading away) in Russia's communication with the West until about the mid-2000s. Then differences emerged, growing increasingly manifest and eventually leading to complete ideological and political disengagement in the middle of the last decade.

The problem in the current dialogue with European partners is that they are following this track by inertia, although no one any longer believes that it will lead somewhere. There is no and won't be any other option in the foreseeable future, since the possibility of rethinking, at least in Europe, is blocked by the confusion within the European
Union itself. This explains why there is such an astonishingly scarce variety of policy measures.

The first reaction to any dissension is a threat of sanctions, with the EU then dancing around it and eventually coming up with purely symbolic measures. They are symbolic because a real revision of relations towards downright confrontation is still problematic due to economic interests, which remain quite strong, and the lack of consensus within the EU. It is noteworthy that the modesty of real measures is made up for by the most confrontational way of their presentation, which clearly poisons the atmosphere further.

The situation with the second model is slightly more complex. The sober-minded part of the political establishment naturally understands that some topics require a purely practical approach, not an ideological one. In fact, relations in the field of nuclear weapons and other ways of inflicting serious military damage upon each other need a pragmatic approach. The military remain sensible in this respect, but there is no such guarantee when it comes to politics. Still, even when professionals understand the importance of discussion and know what to discuss, internal political turmoil can intervene at any time. In this sense, the previous U.S. administration outmatched everybody, holding foreign policy, including the most 


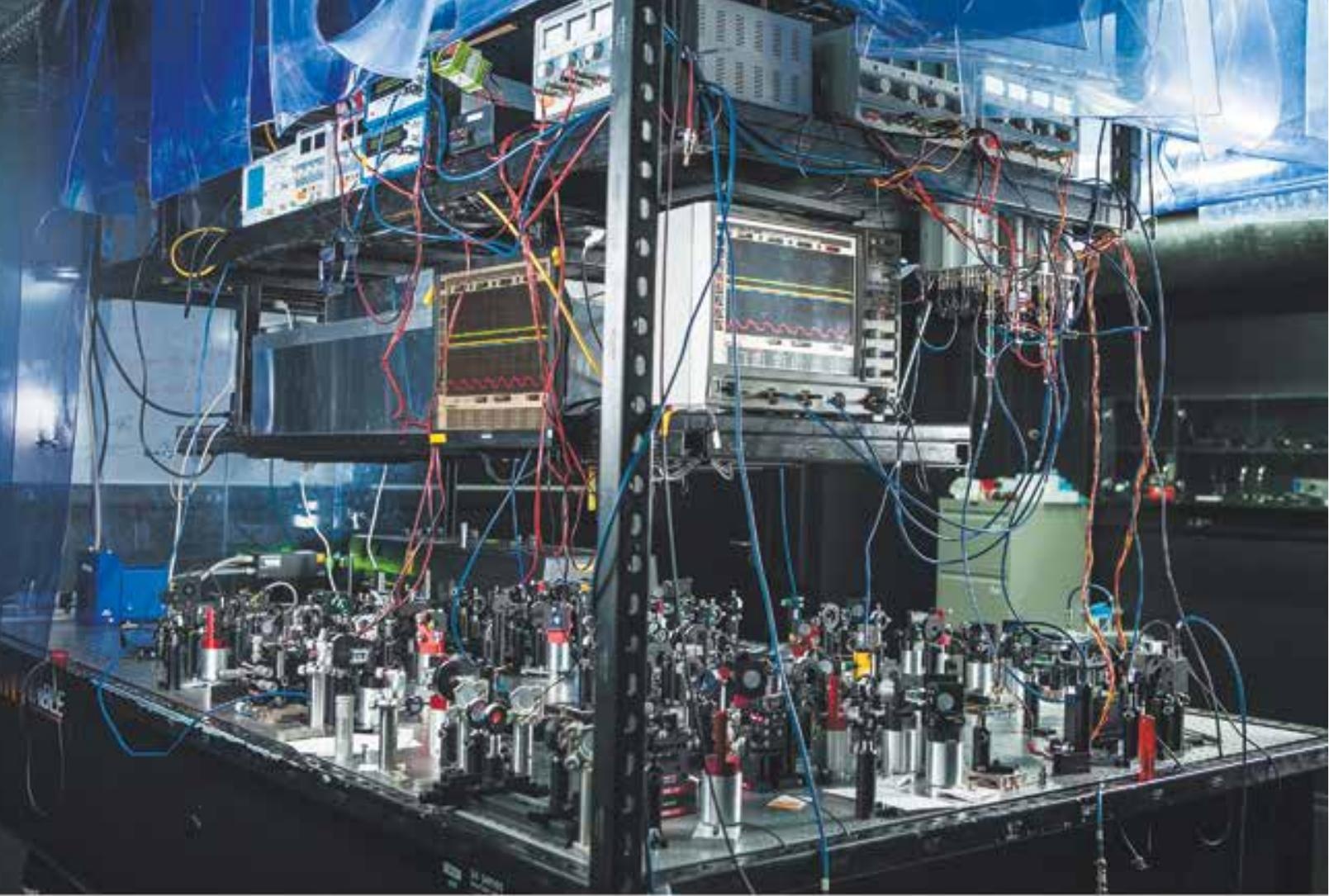

Quantum technologies embodied in microchips and lasers have already changed our world at least once. Now they are about to change it again by controlling matter and light at the level of individual particles. The second quantum revolution will be new materials and ultra-secure communications, superlative accuracy in measurement and efficiency in calculation. The Russian Quantum Center is steadily riding on the crest of this new technological wave.

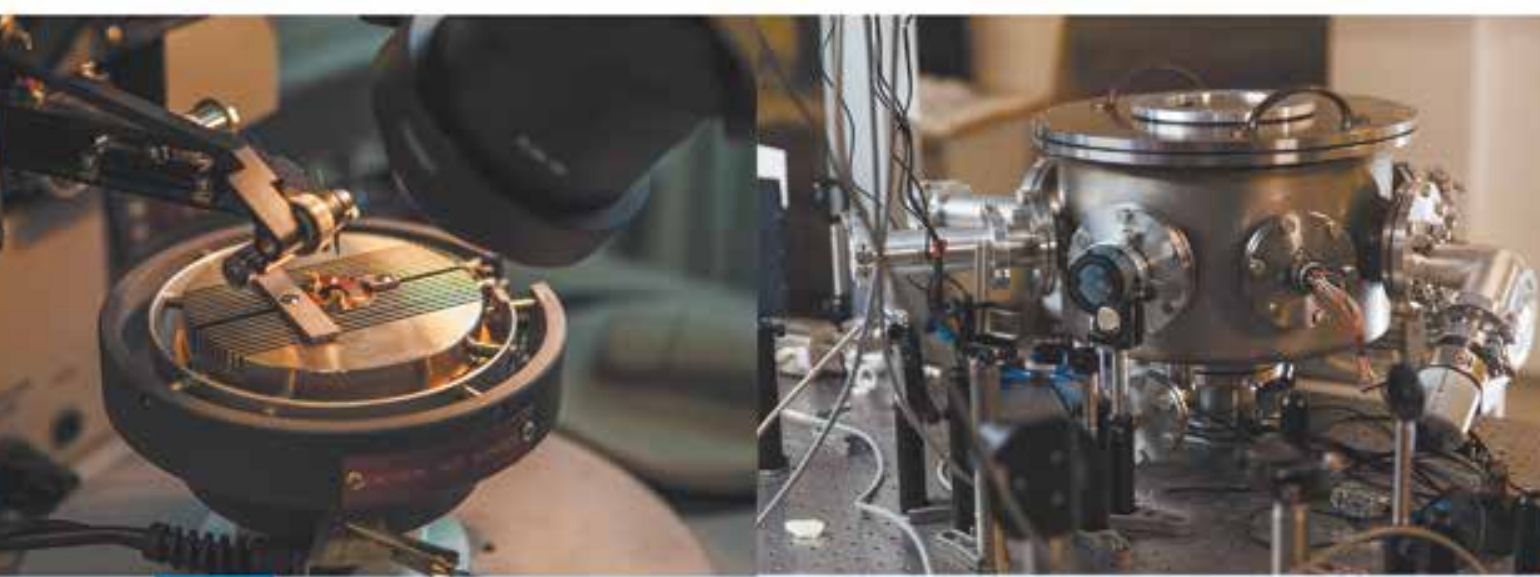


important strategic sphere, hostage to the internal struggle. So, it was practically useless to conduct international dialogue with it. The present American administration is more traditional in this regard, which gives it credit, but the threat of ideological factors interfering where they only spoil things does not disappear even for a second.

The diktat of internal issues also affects the quality of expert or informal dialogue. Reticence and mandatory mantras have always been in use, but now they are forcing everything else out so that the distinction between public and non-public forms of communication is vanishing. This distinction has always been there because contacts become purely decorative unless there is some degree of frankness on the sidelines. This is, among other things, the product of universal exaltation and polarization of positions.

So, all of the above makes the work of experts to establish international ties not so much unnecessary as boring. Everything becomes predictable. The pandemic has reduced personal communication, but, hon- estly, when it does happen, one can hardly notice any freshness or pleasure from it. It seems clear that both the narratives and the tools need to be changed, but no one has the will to do so.

Importantly, the aforesaid concerns the West, but there is never a dull moment with the East. A real meaningful dialogue with Chinese colleagues is just beginning. Previously, they always wore the mask of politeness, evading the discussion of problems, but now the situation forces them to talk to the point. Practical issues are discussed without unnecessary formalities with colleagues from Central Asia. There is a search for topics and forms of dialoguewhich can hardly go on without very concrete discussions - with the Turks and representatives of Arab countries and East Asia. This does not mean that these discussions are easy and pleasant, but they are void of ideological bias, which, unfortunately, has become a trademark of relations with Europe and the United States. Instead, there is a desire to achieve a result. We will come to that with the West, too. In due course. 